International Journal of Pure and Applied Mathematics

Volume 109 No. 3 2016, 583-600

ISSN: 1311-8080 (printed version); ISSN: 1314-3395 (on-line version)

url: http://www.ijpam.eu

doi: 10.12732/ijpam.v109i3.8

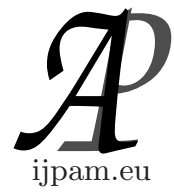

\title{
$\psi-\phi$ CONTRACTION ON SUZUKI TYPE UNIQUE COMMON COUPLED FIXED POINT THEOREM IN PARTIALLY ORDERED MULTIPLICATIVE METRIC SPACES
}

\author{
V.M.L. Hima Bindu ${ }^{1} \S$, G.N.V. Kishore ${ }^{2}$, K.P.R. Rao ${ }^{3}$ \\ ${ }^{1,2}$ Department of Mathematics \\ K.L. University \\ Vaddeswaram, Guntur, 522 502, Andhra Pradesh, INDIA \\ ${ }^{3}$ Department of Mathematics \\ Acharya Nagarjuna University \\ Nagarjuna Nagar, Guntur, 522 510, Andhra Pradesh, INDIA
}

\begin{abstract}
In this paper, we obtain a Suzuki type unique common coupled fixed point theorem by using $\psi-\phi$ contraction in partially ordered multiplicative metric spaces. We also give an example to illustrate our main theorem.
\end{abstract}

AMS Subject Classification: 54H25, 47H10, 54E50

Key Words: multiplicative metric space, $w$-compatible maps, Suzuki type contraction

\section{Introduction}

The notion of multiplicative metric was introduced by Bashirov et al. [3] in 2008. After that Ozavsar and Cevikel[8] investigated its topological properties and proved some fixed point theorems. For more works on fixed, common fixed

\footnotetext{
Received: $\quad$ May 10, 2016

Revised: July 22, 2016

Published: September 30, 2016

(C) 2016 Academic Publications, Ltd.

url: www.acadpubl.eu

$\S_{\text {Correspondence author }}$
} 
point theorems in Multiplicative metric spaces, we refer [5, 2, 6, 9].

The Coupled fixed point is introduced by Bhaskar and Lakshmikantham [4]. Later some of authors proved coupled fixed and coupled common fixed point theorems (See $[1,7,10,11,12])$.

The aim of this paper is to prove Suzuki type unique common coupled fixed point theorem for Jungck type maps by using $\psi-\phi$ contraction condition in partially ordered multiplicative metric spaces.

First we give the following theorem of Suzuki [13].

Theorem 1.1. (See [13]): Let $(X, d)$ be a complete metric space and let $T$ be a mapping on $X$. Define a non-increasing function $\theta:[0,1) \rightarrow\left(\frac{1}{2}, 1\right]$ by

$$
\theta(r)=\left\{\begin{array}{cc}
1 & \text { if } 0 \leq r \leq \frac{(\sqrt{5}-1)}{2} \\
(1-r) r^{-2} & \text { if } \frac{(\sqrt{5}-1)}{2} \leq r \leq \quad 2^{-\frac{1}{2}} \\
(1+r)^{-1} & \text { if } 2^{-\frac{1}{2}} \leq r<1
\end{array}\right.
$$

Assume that there exists $r \in[0,1)$ such that

$$
\theta(r) d(x, T x) \leq d(x, y) \Rightarrow d(T x, T y) \leq r d(x, y)
$$

for all $x, y \in X$. Then there exists a unique fixed point $z$ of $T$. Moreover $\lim _{n \rightarrow \infty} T^{n} x=z$ for all $x \in X$.

Now we recall some basic definitions and examples in multiplicative metric spaces.

Definition 1.2. (See[3]) Multiplicative metric on a nonempty set $X$ is a mapping $d: X \times X \rightarrow R^{+}$satisfying the following conditions:

$\left(d_{1}\right) d(x, y) \geq 1$ for all $x, y \in X$

$\left(d_{2}\right) d(x, y)=1$ if and only if $x=y$,

$\left(d_{3}\right) d(x, y)=d(y, x)$,

$\left(d_{4}\right) d(x, y) \leq d(x, z) \cdot d(z, y)$ for all $x, y, z \in X$.

The pair $(X, d)$ is called a multiplicative metric space.

Example 1.3. (See[9] ) Let $R_{+}^{n}$ be the collection of all $\mathrm{n}$ - tupples of positive real numbers. And let $d^{*}: R_{+}^{n} \times R_{+}^{n} \rightarrow R$ be defined as

$$
d^{*}(x, y)=\left|\frac{x_{1}}{y_{1}}\right|^{*} \cdot\left|\frac{x_{2}}{y_{2}}\right|^{*} \ldots\left|\frac{x_{n}}{y_{n}}\right|^{*}
$$


where $x=\left(x_{1}, x_{2} \cdots x_{n}\right), y=\left(y_{1}, y_{2} \cdots y_{n}\right) \in R_{+}^{n}$ and $|.|^{*}: R_{+} \rightarrow R_{+}$is defined as follows

$$
|a|^{*}=\left\{\begin{array}{lll}
a & \text { if } & a \geq 1 \\
\frac{1}{a} & \text { if } & a<1
\end{array}\right.
$$

Then clearly $d^{*}(x, y)$ is a multiplicative metric.

Example 1.4. (See[9]) Let $d: R \times R \rightarrow[1, \infty)$ be defined as $d(x, y)=$ $a^{|x-y|}$ where $x, y \in R$ and $a>1$. Then $d(x, y)$ is multiplicative metric.

Definition 1.5. (See[8]) Let $(X, d)$ be a multiplicative metric space, $x_{0}$ an arbitrary point in $X$, and $\epsilon>1$. A multiplicative open ball $B\left(x_{0}, \epsilon\right)$ of radius $\epsilon$ centered at $x_{0}$ is the set $\left\{z \in X: d\left(z, x_{0}\right)<\epsilon\right\}$.

A sequence $\left\{x_{n}\right\}$ in a multiplicative metric space $(X, d)$ is said to be multiplicative convergent to some point $x \in X$ if, for any given $\epsilon>1$, then there exists $n_{0} \in N$ such that $x_{n} \in B(x, \epsilon)$ for all $n \geq n_{0}$. If $\left\{x_{n}\right\}$ converges to $x$, we write $x_{n} \rightarrow x$ as $n \rightarrow \infty$.

Definition 1.6. (See[8]) A sequence $\left\{x_{n}\right\}$ in a multiplicative metric space $(X, d)$ is said to be multiplicative convergent to $x$ in $X$ if and only if $d\left(x_{n}, x\right) \rightarrow 1$ as $n \rightarrow \infty$.

Definition 1.7. (See $[8])$ Let $\left(X, d_{X}\right)$ and $\left(Y, d_{Y}\right)$ be two multiplicative metric space and $x_{0}$ an arbitrary but fixed element of $X$. A mapping $f: X \rightarrow Y$ is said to be multiplicative continuous at $x_{0}$ if and only if $x_{n} \rightarrow x_{0}$ in $\left(X, d_{X}\right)$ implies that $f\left(x_{n}\right) \rightarrow f\left(x_{0}\right)$ in $\left(Y, d_{Y}\right)$. That is, given arbitrary $\epsilon>1$, then there exists $\delta>1$ which depends on $x_{0}$ and $\epsilon$ such that $d_{Y}\left(f x, f x_{0}\right)<\epsilon$ for all those $x$ in $X$ for which $d_{X}\left(x, x_{0}\right)<\delta$.

Definition 1.8. (See[8] ) A sequence $\left\{x_{n}\right\}$ in a multiplicative metric space $(X, d)$ is said to be multiplicative Cauchy sequence if, for any $\epsilon>1$, there exists $n_{0} \in N$ such that $d\left(x_{m}, x_{n}\right)<\epsilon$ for all $m, n \geq n_{0}$.

Definition 1.9. (See[8] ) A multiplicative metric space $(X, d)$ is said to be complete if every multiplicative Cauchy sequence $\left\{x_{n}\right\}$ in $X$ is multiplicative convergent in $X$.

Definition 1.10. (See [2]) Let $\Psi$ be the set of all control functions $\varphi$ : $[1, \infty) \rightarrow[1, \infty)$ such that

(i) $\varphi$ is continuous and non - decreasing.

(ii) $\varphi(t)=1$ if and only if $t=1$.

Definition 1.11. [4] An element $(x, y) \in X \times X$ is called a coupled fixed point of mapping $F: X \times X \rightarrow X$ if $x=F(x, y)$ and $y=F(y, x)$. 
Definition 1.12. [1] An element $(x, y) \in X \times X$ is called $\left(g_{1}\right)$ a coupled coincident point of mappings $F: X \times X \rightarrow X$ and $f: X \rightarrow X$ if $f x=F(x, y)$ and $f y=F(y, x)$.

$\left(g_{2}\right)$ a common coupled fixed point of mappings $F: X \times X \rightarrow X$ and $f: X \rightarrow X$ if $x=f x=F(x, y)$ and $y=f y=F(y, x)$.

Definition 1.13. [1] The mappings $F: X \times X \rightarrow X$ and $f: X \rightarrow X$ are called $w$ - compatible if $f(F(x, y))=F(f x, f y)$ and $f(F(y, x))=F(f y, f x)$ whenever $f x=F(x, y)$ and $f y=F(y, x)$.

\section{Main Result}

Theorem 2.1. Let $(X, d, \preceq)$ be partially ordered multiplicative metric space and let $T: X \times X \rightarrow X$ and $g: X \rightarrow X$ be mappings satisfying

$\frac{1}{2} \min \{d(g x, T(x, y)), d(g u, T(u, v))\} \leq \max \{d(g x, g u), d(g y, g v)\}$

implies that $\psi(d(T(x, y), T(u, v))) \leq \frac{\psi(M(x, y, u, v))}{\phi(M(x, y, u, v))}$, for all $x, y, u, v$ in $X$ ,with $g x \preceq g u$ and $g y \succeq g v$ where $\psi, \phi \in \Psi$ and

$$
M(x, y, u, v)=\max \left\{\begin{array}{c}
d(g x, g u), d(g y, g v), d(g x, T(x, y)), \\
d(g y, T(y, x)), d(g u, T(u, v)), d(g v, T(v, u)), \\
\sqrt{d(g x, T(u, v)) \cdot d(g u, T(x, y))}, \\
\sqrt{d(g y, T(v, u)) \cdot d(g v, T(y, x))}
\end{array}\right\}
$$

(2.1.2) $T(X \times X) \subseteq g(X)$ and $g(X)$ is a complete subspaces of $X$,

(2.1.3) $T$ has a mixed $g$ - monotone property

(2.1.4) (a) If a non- decreasing sequence $\left\{x_{n}\right\} \rightarrow x$ then $x_{n} \preceq x$ for all $n$,

(b) If a non- increasing sequence $\left\{y_{n}\right\} \rightarrow y$ then $y \preceq y_{n}$ for all $n$.

If there exist $x_{0}, y_{0} \in X$ such that $g x_{0} \preceq T\left(x_{0}, y_{0}\right)$ and $g y_{0} \succeq T\left(y_{0}, x_{0}\right)$, then $T$ and $g$ have a coupled coincidence point in $X \times X$.

Proof. Let $x_{0}, y_{0} \in X$ such that $g x_{0} \preceq T\left(x_{0}, y_{0}\right)$ and $g y_{0} \succeq T\left(y_{0}, x_{0}\right)$.

Since $T(X \times X) \subseteq g(X)$, we choose $x_{1}, y_{1} \in X$ such that

$$
g x_{0} \preceq T\left(x_{0}, y_{0}\right)=g x_{1}
$$

and

$$
g y_{0} \succeq T\left(y_{0}, x_{0}\right)=g y_{1}
$$


and choose $x_{2}, y_{2} \in X$ such that $g x_{2}=T\left(x_{1}, y_{1}\right)$ and $g y_{2}=T\left(y_{1}, x_{1}\right)$.

Since $T$ has mixed $g$ - monotone property, we obtain $g x_{0} \preceq g x_{1} \preceq g x_{2}$ and $g y_{0} \succeq g y_{1} \succeq g y_{2}$.

Continuing this process, we can construct the sequences $\left\{x_{n}\right\}$ and $\left\{y_{n}\right\}$ in $X$ such that

$$
g x_{n+1}=T\left(x_{n}, y_{n}\right) \quad \text { and } \quad g y_{n+1}=T\left(y_{n}, x_{n}\right), \quad n=0,1,2, \cdots
$$

with

$$
g x_{0} \preceq g x_{1} \preceq g x_{2} \cdots
$$

and

$$
g y_{0} \succeq g y_{1} \succeq g y_{2} \cdots
$$

Case (a) : If $g x_{m}=g x_{m+1}$ and $g y_{m}=g y_{m+1}$ for some $m$.

Then $\left(x_{m}, y_{m}\right)$ is coupled coincidence point in $X \times X$.

Case (b): Assume $g x_{n} \neq g x_{n+1}$ or $g y_{n} \neq g y_{n+1}$ for all $n$.

Since $g x_{n} \preceq g x_{n+1}$ and $g y_{n} \succeq g y_{n+1}$, clearly we have

$$
\begin{aligned}
\frac{1}{2} d\left(g x_{n}, T\left(x_{n}, y_{n}\right)\right) & \leq d\left(g x_{n}, g x_{n+1}\right) \\
& \leq \max \left\{\begin{array}{c}
d\left(g x_{n}, g x_{n+1}\right) \\
d\left(g y_{n}, g y_{n+1}\right)
\end{array}\right\} .
\end{aligned}
$$

Thus

$$
\frac{1}{2} \min \left\{\begin{array}{c}
d\left(g x_{n}, T\left(x_{n}, y_{n}\right)\right), \\
d\left(g x_{n+1}, T\left(x_{n+1}, y_{n+1}\right)\right)
\end{array}\right\} \leq \max \left\{\begin{array}{c}
d\left(g x_{n}, g x_{n+1}\right) \\
d\left(g y_{n}, g y_{n+1}\right)
\end{array}\right\} .
$$

From (2.1.1), we get

$$
\psi\left(d\left(T\left(x_{n}, y_{n}\right), T\left(x_{n+1}, y_{n+1}\right)\right)\right) \leq \frac{\psi\left(M\left(x_{n}, y_{n}, x_{n+1}, y_{n+1}\right)\right)}{\phi\left(M\left(x_{n}, y_{n}, x_{n+1}, y_{n+1}\right)\right)},
$$

where

$$
\begin{aligned}
& M\left(x_{n}, y_{n}, x_{n+1}, y_{n+1}\right) \\
& =\max \left\{\begin{array}{c}
d\left(g x_{n}, g x_{n+1}\right), d\left(g y_{n}, g y_{n+1}\right), \\
d\left(g x_{n}, T\left(x_{n}, y_{n}\right)\right), d\left(g y_{n}, T\left(y_{n}, x_{n}\right)\right), \\
d\left(g x_{n+1}, T\left(x_{n+1}, y_{n+1}\right)\right), d\left(g y_{n+1}, T\left(y_{n+1}, x_{n+1}\right)\right), \\
\sqrt{d\left(g x_{n}, T\left(x_{n+1}, y_{n+1}\right)\right) \cdot d\left(g x_{n+1}, T\left(x_{n}, y_{n}\right)\right)}, \\
\sqrt{d\left(g y_{n}, T\left(y_{n+1}, x_{n+1}\right)\right) \cdot d\left(g y_{n+1}, T\left(y_{n}, x_{n}\right)\right)}
\end{array}\right\}
\end{aligned}
$$




$$
\begin{gathered}
=\max \left\{\begin{array}{c}
d\left(g x_{n}, g x_{n+1}\right), d\left(g y_{n}, g y_{n+1}\right), \\
d\left(g x_{n}, g x_{n+1}\right), d\left(g y_{n}, g y_{n+1}\right), \\
d\left(g x_{n+1}, g x_{n+2}\right), d\left(g y_{n+1}, g y_{n+2}\right), \\
\sqrt{d\left(g x_{n}, g x_{n+2}\right) \cdot d\left(g x_{n+1}, g x_{n+1}\right)}, \\
\sqrt{d\left(g y_{n}, g y_{n+2}\right) \cdot d\left(g y_{n+1}, g y_{n+1}\right)}
\end{array}\right\} \\
=\max \left\{\begin{array}{c}
d\left(g x_{n}, g x_{n+1}\right), d\left(g y_{n}, g y_{n+1}\right), \\
d\left(g x_{n+1}, g x_{n+2}\right), d\left(g y_{n+1}, g y_{n+2}\right), \\
\sqrt{d\left(g x_{n}, g x_{n+2}\right)}, \sqrt{d\left(g y_{n}, g y_{n+2}\right)}
\end{array}\right\} .
\end{gathered}
$$

But

$$
\begin{aligned}
\sqrt{d\left(g x_{n}, g x_{n+2}\right)} & \leq \sqrt{d\left(g x_{n}, g x_{n+1}\right) \cdot d\left(g x_{n+1}, g x_{n+2}\right)} \\
& \leq \max \left\{d\left(g x_{n}, g x_{n+1}\right), d\left(g x_{n+1}, g x_{n+2}\right)\right\}
\end{aligned}
$$

Similarly

$$
\sqrt{d\left(g y_{n}, g y_{n+2}\right)} \leq \max \left\{d\left(g y_{n}, g y_{n+1}\right), d\left(g y_{n+1}, g y_{n+2}\right)\right\}
$$

Therefore

$$
M\left(x_{n}, y_{n}, x_{n+1}, y_{n+1}\right)=\max \left\{\begin{array}{c}
d\left(g x_{n}, g x_{n+1}\right), d\left(g y_{n}, g y_{n+1}\right), \\
d\left(g x_{n+1}, g x_{n+2}\right), d\left(g y_{n+1}, g y_{n+2}\right)
\end{array}\right\} .
$$

Put $R_{n}=\max \left\{d\left(g x_{n}, g x_{n+1}\right), d\left(g y_{n}, g y_{n+1}\right)\right\}$.

Hence

$$
\psi\left(d\left(g x_{n+1}, g x_{n+2}\right)\right) \leq \frac{\psi\left(\max \left\{R_{n}, R_{n+1}\right\}\right)}{\phi\left(\max \left\{R_{n}, R_{n+1}\right\}\right)} .
$$

Similarly

$$
\psi\left(d\left(g y_{n+1}, g y_{n+2}\right)\right) \leq \frac{\psi\left(\max \left\{R_{n}, R_{n+1}\right\}\right)}{\phi\left(\max \left\{R_{n}, R_{n+1}\right\}\right)}
$$

Now

$$
\begin{aligned}
\psi\left(R_{n+1}\right) & =\psi\left(\max \left\{d\left(g x_{n+1}, g x_{n+2}\right), d\left(g y_{n+1}, g y_{n+2}\right)\right\}\right) \\
& =\max \left\{\psi\left(d\left(g x_{n+1}, g x_{n+2}\right)\right), \psi\left(d\left(g y_{n+1}, g y_{n+2}\right)\right)\right\} \\
& \leq \frac{\psi\left(\max \left\{R_{n}, R_{n+1}\right\}\right)}{\phi\left(\max \left\{R_{n}, R_{n+1}\right\}\right)} .
\end{aligned}
$$


If $R_{n+1}$ is maximum, we get

$$
\psi\left(R_{n+1}\right) \leq \frac{\psi\left(R_{n+1}\right)}{\phi\left(R_{n+1}\right)}<\psi\left(R_{n+1}\right)
$$

which is a contradiction.

Hence $R_{n}$ is a maximum

$$
\begin{aligned}
\psi\left(R_{n+1}\right) & \leq \frac{\psi\left(R_{n}\right)}{\phi\left(R_{n}\right)} \\
& <\psi\left(R_{n}\right)
\end{aligned}
$$

Since $\psi$ is non - decreasing, we obtain

$$
R_{n+1} \leq R_{n}
$$

Thus $\left\{R_{n}\right\}$ is non- increasing sequence of non- negative real number and must converges to a real number $r \geq 1$ (say).

Suppose $r>1$.

Letting $n \rightarrow \infty$ in (1), we get

$$
\psi(r) \leq \frac{\psi(r)}{\phi(r)}<\psi(r)
$$

which is a contradiction.

Hence $r=1$. Thus

$$
\lim _{n \rightarrow \infty} \max \left\{d\left(g x_{n}, g x_{n+1}\right), d\left(g y_{n}, g y_{n+1}\right)\right\}=1
$$

which implies that

$$
\lim _{n \rightarrow \infty} d\left(g x_{n}, g x_{n+1}\right)=1=\lim _{n \rightarrow \infty} d\left(g y_{n}, g y_{n+1}\right) .
$$

Now we prove that $\left\{g x_{n}\right\}$ and $\left\{g y_{n}\right\}$ are multiplicative Cauchy sequences.

On contrary suppose that $\left\{g x_{n}\right\}$ or $\left\{g y_{n}\right\}$ are not multiplicative Cauchy sequences.

Then there exist an $\epsilon>1$ and monotone increasing sequence of natural numbers $\left\{m_{k}\right\}$ amd $\left\{n_{k}\right\}$ such that $n_{k}>m_{k}>k$,

$$
\max \left\{d\left(g x_{m_{k}}, g x_{n_{k}}\right), d\left(g y_{m_{k}}, g y_{n_{k}}\right)\right\} \geq \epsilon
$$

and

$$
\max \left\{d\left(g x_{m_{k}}, g x_{n_{k-1}}\right), d\left(g y_{m_{k}}, g y_{n_{k-1}}\right)\right\}<\epsilon
$$


From (3) and (4), we have

$$
\begin{aligned}
\epsilon & \leq \max \left\{d\left(g x_{m_{k}}, g x_{n_{k}}\right), d\left(g y_{m_{k}}, g y_{n_{k}}\right)\right\} \\
& \leq \max \left\{d\left(g x_{m_{k}}, g x_{n_{k-1}}\right) \cdot d\left(g x_{n_{k-1}}, g x_{n_{k}}\right), d\left(g y_{m_{k}}, g y_{n_{k-1}}\right) \cdot d\left(g y_{n_{k-1}}, g y_{n_{k}}\right)\right\} .
\end{aligned}
$$

Letting $k \rightarrow \infty$, we have

$$
\epsilon \leq \lim _{k \rightarrow \infty} \max \left\{d\left(g x_{m_{k}}, g x_{n_{k}}\right), d\left(g y_{m_{k}}, g y_{n_{k}}\right)\right\} \leq \epsilon
$$

Thus

$$
\lim _{k \rightarrow \infty} \max \left\{d\left(g x_{m_{k}}, g x_{n_{k}}\right), d\left(g y_{m_{k}}, g y_{n_{k}}\right)\right\}=\epsilon .
$$

Form (3), we have

$$
\begin{aligned}
\epsilon & \leq \max \left\{d\left(g x_{m_{k}}, g x_{n_{k}}\right), d\left(g y_{m_{k}}, g y_{n_{k}}\right)\right\} \\
& \leq \max \left\{d\left(g x_{m_{k}}, g x_{n_{k+1}}\right) \cdot d\left(g x_{n_{k+1}}, g x_{n_{k}}\right), d\left(g y_{m_{k}}, g y_{n_{k+1}}\right) \cdot d\left(g y_{n_{k+1}}, g y_{n_{k}}\right)\right\} \\
& \leq \max \left\{\begin{array}{c}
\left(d\left(g x_{m_{k}}, g x_{n_{k}}\right) \cdot d\left(g x_{n_{k}}, g x_{n_{k+1}}\right) \cdot d\left(g x_{n_{k+1}}, g x_{n_{k}}\right),\right. \\
d\left(g y_{m_{k}}, g y_{n_{k}}\right) \cdot d\left(g y_{n_{k}}, g y_{n_{k+1}}\right) \cdot d\left(g y_{n_{k+1}}, g y_{n_{k}}\right)
\end{array}\right\} .
\end{aligned}
$$

Letting $k \rightarrow \infty$, we have

$$
\lim _{k \rightarrow \infty} \max \left\{d\left(g x_{m_{k}}, g x_{n_{k+1}}\right), d\left(g y_{m_{k}}, g y_{n_{k+1}}\right)\right\}=\epsilon
$$

From (3), we have

$$
\begin{aligned}
\epsilon & \leq \max \left\{d\left(g x_{m_{k}}, g x_{n_{k}}\right), d\left(g y_{m_{k}}, g y_{n_{k}}\right)\right\} \\
& \leq \max \left\{\begin{array}{c}
d\left(g x_{m_{k}}, g x_{m_{k+1}}\right) \cdot d\left(g x_{m_{k+1}}, g x_{n_{k}}\right), \\
d\left(g y_{m_{k}}, g y_{m_{k+1}}\right) \cdot d\left(g y_{m_{k+1}}, g y_{n_{k}}\right)
\end{array}\right\} \\
& \leq \max \left\{\begin{array}{c}
d\left(g x_{m_{k}}, g x_{m_{k+1}}\right) \cdot d\left(g x_{m_{k+1}}, g x_{n_{k+2}}\right) \cdot d\left(g x_{n_{k+2}}, g x_{n_{k}}\right), \\
d\left(g y_{m_{k}}, g y_{m_{k+1}}\right) \cdot d\left(g y_{m_{k+1}}, g y_{n_{k+2}}\right) \cdot d\left(g y_{n_{k+2}}, g y_{n_{k}}\right)
\end{array}\right\} \\
& \leq \max \left\{\begin{array}{c}
d\left(g x_{m_{k}}, g x_{m_{k+1}}\right) \cdot d\left(g x_{m_{k+1}}, g x_{n_{k+2}}\right) \cdot d\left(g x_{n_{k+2}}, g x_{n_{k+1}}\right) \cdot d\left(g x_{n_{k+1}}, g x_{n_{k}}\right), \\
d\left(g y_{m_{k}}, g y_{m_{k+1}}\right) \cdot d\left(g y_{m_{k+1}}, g y_{n_{k+2}}\right) \cdot d\left(g y_{n_{k+2}}, g y_{n_{k+1}}\right) \cdot d\left(g y_{n_{k+1}}, g y_{n_{k}}\right)
\end{array}\right\} \\
& \leq \max \left\{\begin{array}{c}
\left(d\left(g x_{m_{k}}, g x_{m_{k+1}}\right)\right)^{2} \cdot d\left(g x_{m_{k}}, g x_{n_{k}}\right) \cdot\left(d\left(g x_{n_{k+2}}, g x_{n_{k+1}}\right)\right)^{2} \cdot\left(d\left(g x_{n_{k+1}}, g x_{n_{k}}\right)\right)^{2}, \\
\left(d\left(g y_{m_{k}}, g y_{m_{k+1}}\right)\right)^{2} \cdot d\left(g y_{m_{k}}, g y_{n_{k}}\right) \cdot\left(d\left(g y_{n_{k+2}}, g y_{n_{k+1}}\right)\right)^{2} \cdot\left(d\left(g y_{n_{k+1}}, g y_{n_{k}}\right)\right)^{2}
\end{array}\right\} .
\end{aligned}
$$

Letting $k \rightarrow \infty$, using (2), (5) and (7), we have

$$
\lim _{k \rightarrow \infty} \max \left\{d\left(g x_{m_{k}+1}, g x_{n_{k+2}}\right), d\left(g y_{m_{k}+1}, g y_{n_{k+2}}\right)\right\}=\epsilon .
$$

Also from (3), we have

$$
\epsilon \leq \max \left\{d\left(g x_{m_{k}}, g x_{n_{k}}\right), d\left(g y_{m_{k}}, g y_{n_{k}}\right)\right\}
$$




$$
\begin{aligned}
& \leq \max \left\{\begin{array}{c}
d\left(g x_{m_{k}}, g x_{n_{k+2}}\right) \cdot d\left(g x_{n_{k+2}}, g x_{n_{k}}\right), \\
d\left(g y_{m_{k}}, g y_{n_{k+2}}\right) \cdot d\left(g y_{n_{k+2}}, g y_{n_{k}}\right)
\end{array}\right\} \\
& \leq \max \left\{\begin{array}{c}
d\left(g x_{m_{k}}, g x_{n_{k+2}}\right) \cdot d\left(g x_{n_{k}+2}, g x_{n_{k+1}}\right) \cdot d\left(g x_{n_{k+1}}, g x_{n_{k}}\right), \\
d\left(g y_{m_{k}}, g y_{n_{k+2}}\right) \cdot d\left(g y_{n_{k}+2}, g y_{n_{k+1}}\right) \cdot d\left(g y_{n_{k+1}}, g y_{n_{k}}\right)
\end{array}\right\} \\
& \leq \max \left\{\begin{array}{c}
d\left(g x_{m_{k}}, g x_{n_{k}}\right) \cdot\left(d\left(g x_{n_{k}+2}, g x_{n_{k+1}}\right)\right)^{2} \cdot\left(d\left(g x_{n_{k+1}}, g x_{n_{k}}\right)\right)^{2}, \\
d\left(g y_{m_{k}}, g y_{n_{k}}\right) \cdot\left(d\left(g y_{n_{k}+2}, g y_{n_{k+1}}\right)\right)^{2} \cdot\left(d\left(g y_{n_{k+1}}, g y_{n_{k}}\right)\right)^{2}
\end{array}\right\} .
\end{aligned}
$$

Letting $k \rightarrow \infty$, from (2),(5) and (9)

$$
\lim _{k \rightarrow \infty} \max \left\{d\left(g x_{m_{k}}, g x_{n_{k+2}}\right), d\left(g y_{m_{k}}, g y_{n_{k+2}}\right)\right\}=\epsilon .
$$

Again from (3), we have

$$
\begin{aligned}
\epsilon & \leq \max \left\{d\left(g x_{m_{k}}, g x_{n_{k}}\right), d\left(g y_{m_{k}}, g y_{n_{k}}\right)\right\} \\
& \leq \max \left\{\begin{array}{c}
d\left(g x_{m_{k}}, g x_{m_{k+1}}\right) \cdot d\left(g x_{m_{k+1}}, g x_{n_{k}}\right), \\
d\left(g y_{m_{k}}, g y_{m_{k+1}}\right) \cdot d\left(g y_{n_{k+1}}, g y_{n_{k}}\right)
\end{array}\right\} \\
& \leq \max \left\{\begin{array}{c}
d\left(g x_{m_{k}}, g x_{m_{k+1}}\right) \cdot d\left(g x_{m_{k}+1}, g x_{n_{k+1}}\right) \cdot d\left(g x_{n_{k+1}}, g x_{n_{k}}\right), \\
d\left(g y_{m_{k}}, g y_{m_{k+1}}\right) \cdot d\left(g y_{m_{k}+1}, g y_{n_{k+1}}\right) \cdot d\left(g y_{n_{k+1}}, g y_{n_{k}}\right)
\end{array}\right\} \\
& \leq \max \left\{\begin{array}{c}
\left(d\left(g x_{m_{k}}, g x_{m_{k+1}}\right)\right)^{2} \cdot d\left(g x_{m_{k}}, g x_{n_{k}}\right) \cdot\left(d\left(g x_{n_{k+1}}, g x_{n_{k}}\right)\right)^{2}, \\
\left(d\left(g y_{m_{k}}, g y_{m_{k+1}}\right)\right)^{2} \cdot d\left(g y_{m_{k}}, g y_{n_{k}}\right) \cdot\left(d\left(g y_{n_{k+1}}, g y_{n_{k}}\right)\right)^{2}
\end{array}\right\} .
\end{aligned}
$$

Letting $k \rightarrow \infty$, from (2),(5) and (11), we have that

$$
\lim _{k \rightarrow \infty} \max \left\{d\left(g x_{m_{k}+1}, g x_{n_{k+1}}\right), d\left(g y_{m_{k}+1}, g y_{n_{k+1}}\right)\right\}=\epsilon .
$$

Now from (3), we get

$$
\begin{aligned}
\epsilon & \leq \max \left\{d\left(g x_{m_{k}}, g x_{n_{k}}\right), d\left(g y_{m_{k}}, g y_{n_{k}}\right)\right\} \\
& \leq \max \left\{d\left(g x_{m_{k}}, g x_{n_{k+1}}\right) \cdot d\left(g x_{n_{k+1}}, g x_{n_{k}}\right), d\left(g y_{m_{k}}, g y_{n_{k+1}}\right) \cdot d\left(g y_{n_{k+1}}, g y_{n_{k}}\right)\right\} \\
& \leq \max \left\{\begin{array}{c}
d\left(g x_{m_{k}}, g x_{m_{k+1}}\right) \cdot d\left(g x_{m_{k}+1}, g x_{n_{k+2}}\right) \cdot d\left(g x_{n_{k+2}}, g x_{n_{k+1}}\right) \cdot d\left(g x_{n_{k+1}}, g x_{n_{k}}\right), \\
d\left(g y_{m_{k}}, g y_{m_{k+1}}\right) \cdot d\left(g y_{m_{k}+1}, g y_{n_{k+2}}\right) \cdot d\left(g y_{n_{k+2}}, g y_{n_{k+1}}\right) \cdot d\left(g y_{n_{k+1}}, g y_{n_{k}}\right)
\end{array}\right\} .
\end{aligned}
$$

Letting $k \rightarrow \infty$, using (2), we obtain that

$$
\epsilon \leq \lim _{k \rightarrow \infty} \max \left\{d\left(g x_{m_{k+1}}, g x_{n_{k+2}}\right), d\left(g y_{m_{k+1}}, g y_{n_{k+2}}\right)\right\} .
$$

By property of $\psi,(5),(6)$ and (8), we have

$$
\psi(\epsilon) \leq \lim _{k \rightarrow \infty} \psi\left(\max \left\{d\left(g x_{m_{k+1}}, g x_{n_{k+2}}\right), d\left(g y_{m_{k+1}}, g y_{n_{k+2}}\right)\right\}\right)
$$




$$
=\lim _{k \rightarrow \infty} \max \left\{\psi\left(d\left(g x_{m_{k}+1}, g x_{n_{k}+2}\right)\right), \psi\left(d\left(g y_{m_{k}+1}, g y_{n_{k}+2}\right)\right)\right\}
$$

Now we will show that

$$
\begin{array}{r}
\frac{1}{2} \min \left\{\begin{array}{c}
d\left(g x_{m_{k}}, T\left(x_{m_{k}}, y_{m_{k}}\right)\right) \\
d\left(g x_{n_{k}+1}, T\left(x_{n_{k}+1}, y_{n_{k}+1}\right)\right)
\end{array}\right\} \\
\leq \max \left\{d\left(g x_{m_{k}}, g x_{n_{k}+1}\right), d\left(g y_{m_{k}}, g x_{n_{k}+1}\right)\right\}
\end{array}
$$

On contrary suppose that

$$
\begin{aligned}
& \max \left\{d\left(g x_{m_{k}}, g x_{n_{k}+1}\right), d\left(g y_{m_{k}}, g x_{n_{k}+1}\right)\right\} \\
&<\frac{1}{2} \min \left\{\begin{array}{c}
d\left(g x_{m_{k}}, T\left(x_{m_{k}}, y_{m_{k}}\right)\right), \\
d\left(g x_{n_{k}+1}, T\left(x_{n_{k}+1}, y_{n_{k}+1}\right)\right)
\end{array}\right\} .
\end{aligned}
$$

Letting $k \rightarrow \infty$, we have

$$
\epsilon \leq \frac{1}{2} \min \{1,1\}=\frac{1}{2}
$$

which is a contradiction.

Hence

$$
\begin{array}{r}
\frac{1}{2} \min \left\{\begin{array}{c}
d\left(g x_{m_{k}}, T\left(x_{m_{k}}, y_{m_{k}}\right)\right) \\
d\left(g x_{n_{k}+1}, T\left(x_{n_{k}+1}, y_{n_{k}+1}\right)\right)
\end{array}\right\} \\
\leq \max \left\{d\left(g x_{m_{k}}, g x_{n_{k}+1}\right), d\left(g y_{m_{k}}, g x_{n_{k}+1}\right)\right\}
\end{array}
$$

Similarly

$$
\begin{array}{r}
\frac{1}{2} \min \left\{\begin{array}{c}
d\left(g y_{m_{k}}, T\left(y_{m_{k}}, x_{m_{k}}\right)\right) \\
d\left(g y_{n_{k}+1}, T\left(y_{n_{k}+1}, x_{n_{k}+1}\right)\right)
\end{array}\right\} \\
\leq \max \left\{d\left(g x_{m_{k}}, g x_{n_{k}+1}\right), d\left(g y_{m_{k}}, g x_{n_{k}+1}\right)\right\}
\end{array}
$$

Now from (2.1.1), we get

$$
\psi\left(d\left(T\left(x_{m_{k}}, y_{m_{k}}\right), T\left(x_{n_{k}+1}, y_{n_{k}+1}\right)\right)\right) \leq \frac{\psi\left(M\left(x_{m_{k}}, y_{m_{k}}, x_{n_{k}+1}, y_{n_{k}+1}\right)\right)}{\phi\left(M\left(x_{m_{k}}, y_{m_{k}}, x_{n_{k}+1}, y_{n_{k}+1}\right)\right)}
$$

where

$$
M\left(x_{m_{k}}, y_{m_{k}}, x_{n_{k+1}}, y_{n_{k}+1}\right)
$$




$$
\begin{aligned}
& =\max \left\{\begin{array}{c}
d\left(g x_{m_{k}}, g x_{n_{k+1}}\right), d\left(g y_{m_{k}}, g y_{n_{k+1}}\right), \\
d\left(g x_{m_{k}}, T\left(x_{m_{k}}, y_{m_{k}}\right)\right), d\left(g y_{m_{k}}, T\left(y_{m_{k}}, x_{m_{k}}\right)\right), \\
d\left(g x_{n_{k+1}}, T\left(x_{n_{k+1}}, y_{n_{k+1}}\right)\right), d\left(g y_{n_{k+1}}, T\left(y_{n_{k+1}}, x_{n_{k+1}}\right)\right), \\
\sqrt{d\left(g x_{m_{k}}, T\left(x_{n_{k+1}}, y_{n_{k+1}}\right)\right) \cdot d\left(g x_{n_{k+1}}, T\left(x_{m_{k}}, y_{m_{k}}\right)\right)}, \\
\sqrt{d\left(g y_{m_{k}}, T\left(y_{n_{k+1}}, x_{n_{k+1}}\right)\right) \cdot d\left(g y_{n_{k+1}}, T\left(y_{m_{k}}, x_{m_{k}}\right)\right)}
\end{array}\right\} \\
& =\max \left\{\begin{array}{c}
d\left(g x_{m_{k}}, g x_{n_{k+1}}\right), d\left(g y_{m_{k}}, g y_{n_{k+1}}\right), \\
d\left(g x_{m_{k}}, g x_{m_{k}+1}\right), d\left(g y_{m_{k}}, g y_{m_{k}+1}\right), \\
d\left(g x_{n_{k+1}}, g x_{n_{k+2}}\right), d\left(g y_{n_{k+1}}, g y_{n_{k+2}}\right), \\
\sqrt{d\left(g x_{m_{k}}, g x_{n_{k+2}}\right) \cdot d\left(g x_{n_{k+1}}, g x_{m_{k+1}}\right)}, \\
\sqrt{d\left(g y_{m_{k}}, g y_{n_{k+2}}\right) \cdot d\left(g y_{n_{k+1}}, g y_{m_{k+1}}\right)}
\end{array}\right\} .
\end{aligned}
$$

But

$$
\begin{aligned}
& \max \left\{\begin{array}{l}
\sqrt{d\left(g x_{m_{k}}, g x_{n_{k+2}}\right) \cdot d\left(g x_{n_{k+1}}, g x_{m_{k+1}}\right)}, \\
\sqrt{d\left(g y_{m_{k}}, g y_{n_{k+2}}\right) \cdot d\left(g y_{n_{k+1}}, g y_{m_{k+1}}\right)}
\end{array}\right\} \\
& =\max \left\{\begin{array}{c}
\max \left\{\begin{array}{c}
d\left(g x_{m_{k}}, g x_{n_{k+2}}\right), \\
d\left(g x_{n_{k+1}}, g x_{m_{k+1}}\right)
\end{array}\right\}, \\
\max \left\{\begin{array}{c}
d\left(g y_{m_{k}}, g y_{n_{k+2}}\right), \\
d\left(g y_{n_{k+1}}, g y_{m_{k+1}}\right)
\end{array}\right\}
\end{array}\right\} \\
& =\max \left\{\begin{array}{c}
d\left(g x_{m_{k}}, g x_{n_{k+2}}\right), \\
d\left(g x_{n_{k+1}}, g x_{m_{k+1}}\right), \\
d\left(g y_{m_{k}}, g y_{n_{k+2}}\right), \\
d\left(g y_{n_{k+1}}, g y_{m_{k+1}}\right)
\end{array}\right\} \\
& \rightarrow \epsilon \text { as } k \rightarrow \infty \text {. }
\end{aligned}
$$

So that

$$
\lim _{k \rightarrow \infty} M\left(x_{m_{k}}, y_{m_{k}}, x_{n_{k+1}}, y_{n_{k}+1}\right)=\epsilon .
$$

Hence letting $k \rightarrow \infty$ in (14), we have that

$$
\lim _{k \rightarrow \infty} \psi\left(d\left(g x_{m_{k}+1}, g x_{n_{k}+2}\right)\right) \leq \frac{\psi(\epsilon)}{\phi(\epsilon)} .
$$

Similarly

$$
\lim _{k \rightarrow \infty} \psi\left(d\left(g y_{m_{k}+1}, g y_{n_{k}+2}\right)\right) \leq \frac{\psi(\epsilon)}{\phi(\epsilon)}
$$


From (13), we have

$$
\begin{aligned}
\psi(\epsilon) & \leq \frac{\psi(\epsilon)}{\phi(\epsilon)} \\
& <\psi(\epsilon)
\end{aligned}
$$

which is a contradiction.

Hence $\left\{g x_{n}\right\}$ and $\left\{g y_{n}\right\}$ are Cauchy sequences in the multiplicative metric space $(X, d)$.

Hence we have

$$
\lim _{n, m \rightarrow \infty} d\left(g x_{n}, g x_{m}\right)=1=\lim _{n, m \rightarrow \infty} d\left(g y_{n}, g y_{m}\right) .
$$

Suppose $g(X)$ is a complete subspace of $X$.

Since $\left\{g x_{n}\right\}$ and $\left\{g y_{n}\right\}$ are Cauchy sequence in the multiplicative metric space $(g(X), d)$.

It follows that $\left\{g x_{n}\right\}$ and $\left\{g y_{n}\right\}$ convergent to some $\alpha$ and $\beta$ in $g(X)$.

Thus

$$
\lim _{n \rightarrow \infty} d\left(g x_{n}, \alpha\right)=1
$$

and

$$
\lim _{n \rightarrow \infty} d\left(g y_{n}, \beta\right)=1 \text {. }
$$

Since $\alpha, \beta \in g(X)$, there exist $x, y \in X$ such that $\alpha=g x$ and $\beta=g y$.

Since $\left\{g x_{n}\right\}$ is non - decreasing sequence and $\left\{g x_{n}\right\} \rightarrow \alpha=g x$. It follows that $g x_{n+1} \preceq g x=\alpha$.

Also $\left\{g y_{n}\right\}$ is non - increasing sequence and $\left\{g y_{n}\right\} \rightarrow \beta=g y$. It follows that $g y_{n+1} \succeq g y=\beta$.

Now we claim that for each $n \geq 1$, at least one of the following holds

$$
\frac{1}{2} \min \left\{d\left(g x_{n}, g x_{n+1}\right), d(g x, T(x, y))\right\} \leq \max \left\{d\left(g x_{n}, g x\right), d\left(g y_{n}, g y\right)\right\}
$$

or

$$
\begin{aligned}
\frac{1}{2} \min \left\{d\left(g x_{n+1}, g x_{n+2}\right), d(g x, T(x, y))\right\} & \\
& \leq \max \left\{d\left(g x_{n+1}, g x\right), d\left(g y_{n+1}, g y\right)\right\} .
\end{aligned}
$$

On contrary suppose that

$$
\frac{1}{2} \min \left\{d\left(g x_{n}, g x_{n+1}\right), d(g x, T(x, y))\right\}>\max \left\{d\left(g x_{n}, g x\right), d\left(g y_{n}, g y\right)\right\}
$$

and 


$$
\begin{aligned}
\frac{1}{2} \min \left\{d\left(g x_{n+1}, g x_{n+2}\right), d(g x, T(x, y))\right\} & \\
& >\max \left\{d\left(g x_{n+1}, g x\right), d\left(g y_{n+1}, g y\right)\right\} .
\end{aligned}
$$

Now

$$
\begin{aligned}
d\left(g x_{n}, g x_{n+1}\right) & \leq d\left(g x_{n}, g x\right) \cdot d\left(g x, g x_{n+1}\right) \\
& <\frac{1}{2} d\left(g x_{n}, g x_{n+1}\right) \cdot \frac{1}{2} d\left(g x_{n+1}, g x_{n+2}\right) \\
& =\frac{1}{4} d\left(g x_{n}, g x_{n+1}\right) \cdot d\left(g x_{n+1}, g x_{n+2}\right) .
\end{aligned}
$$

Letting $n \rightarrow \infty$ and using (2), we have

$$
1 \leq \frac{1}{4}
$$

which is a contradiction.

Hence claim holds.

Sub case (i) : Suppose

$$
\frac{1}{2} \min \left\{d\left(g x_{n}, T\left(x_{n}, y_{n}\right)\right), d(g x, T(x, y))\right\} \leq \max \left\{d\left(g x_{n}, g x\right), d\left(g y_{n}, g y\right)\right\}
$$

Now we prove that $T(x, y)=\alpha, T(y, x)=\beta$.

From (2.1.1), we get that

$$
\psi\left(d\left(T\left(x_{n}, y_{n}\right), T(x, y)\right)\right) \leq \frac{\psi\left(M\left(x_{n}, y_{n}, x, y\right)\right)}{\phi\left(M\left(x_{n}, y_{n}, x, y\right)\right)}
$$

where

$$
\begin{gathered}
M\left(x_{n}, y_{n}, x, y\right)=\max \left\{\begin{array}{c}
d\left(g x_{n}, g x\right), d\left(g y_{n}, g y\right), d\left(g x_{n}, T\left(x_{n}, y_{n}\right)\right), \\
d\left(g y_{n}, T\left(y_{n}, x_{n}\right)\right), d(g x, T(x, y)), d(g y, T(y, x)), \\
\sqrt{d\left(g x_{n}, T(x, y)\right) \cdot d\left(g x, T\left(x_{n}, y_{n}\right)\right)}, \\
\sqrt{d\left(g y_{n}, T(y, x)\right) \cdot d\left(g y, T\left(y_{n}, x_{n}\right)\right)}
\end{array}\right\} \\
=\max \left\{\begin{array}{c}
d\left(g x_{n}, \alpha\right), d\left(g y_{n}, \beta\right), d\left(g x_{n}, T\left(x_{n}, y_{n}\right)\right), \\
d\left(g y_{n}, T\left(y_{n}, x_{n}\right)\right), d(\alpha, T(x, y)), d(\beta, T(y, x)), \\
\sqrt{d\left(g x_{n}, T(x, y)\right) \cdot d\left(\alpha, T\left(x_{n}, y_{n}\right)\right)}, \\
\sqrt{d\left(g y_{n}, T(y, x)\right) \cdot d\left(\beta, T\left(y_{n}, x_{n}\right)\right)}
\end{array}\right\} .
\end{gathered}
$$


Letting $n \rightarrow \infty$, we have

$$
\begin{aligned}
\lim _{n \rightarrow \infty} M\left(x_{n}, y_{n}, x, y\right) & =\max \left\{\begin{array}{c}
1,1,1,1, d(\alpha, T(x, y)), d(\beta, T(y, x)), \\
\sqrt{d(\alpha, T(x, y))}, \sqrt{d(\beta, T(y, x))}
\end{array}\right\} \\
& =\max \{d(\alpha, T(x, y)), d(\beta, T(y, x))\} .
\end{aligned}
$$

Thus

$$
\psi(d(\alpha, T(x, y))) \leq \frac{\psi(\max \{d(\alpha, T(x, y)), d(\beta, T(y, x))\})}{\phi(\max \{d(\alpha, T(x, y)), d(\beta, T(y, x))\})} .
$$

Similarly we can show that

$$
\psi(d(\beta, T(y, x))) \leq \frac{\psi(\max \{d(\alpha, T(x, y)), d(\beta, T(y, x))\})}{\phi(\max \{d(\alpha, T(x, y)), d(\beta, T(y, x))\})} .
$$

Now consider

$$
\begin{gathered}
\psi(\max \{d(\alpha, T(x, y)), d(\beta, T(y, x))\}) \\
=\max \{\psi(d(\alpha, T(x, y))), \psi(d(\beta, T(y, x)))\} \\
\leq \frac{\psi(\max \{d(\alpha, T(x, y)), d(\beta, T(y, x))\})}{\phi(\max \{d(\alpha, T(x, y)), d(\beta, T(y, x))\})} .
\end{gathered}
$$

Therefore

$$
\phi(\max \{d(\alpha, T(x, y)), d(\beta, T(y, x))\}) \leq 1 .
$$

Thus $\alpha=T(x, y)=g x$ and $\beta=T(y, x)=g y$.

Therefore $(x, y)$ is a coupled coincidence point of $T$ and $g$.

There exist coupled coincidence point of $T$ and $g$ when

$$
\begin{aligned}
\frac{1}{2} \min \left\{d\left(g x_{n+1}, g x_{n+2}\right), d(g x, T(x, y))\right. & \\
& \leq \max \left\{d\left(g x_{n+1}, g x\right), d\left(g y_{n+1}, g y\right)\right\}
\end{aligned}
$$

holds.

Theorem 2.2. In addition to the hypothesis of Theorem 2.1, suppose that for every $(x, y),\left(x^{\prime}, y^{\prime}\right) \in X \times X$, there exists $(u, v) \in X \times X$ such that $(T(u, v), T(v, u))$ is comparable to $(T(x, y), T(y, x))$ and $\left(T\left(x^{\prime}, y^{\prime}\right), T\left(y^{\prime}, x^{\prime}\right)\right)$.

Moreover if $(T, g)$ is $w$ - compatible then $T$ and $g$ have a unique common coupled fixed point in $X \times X$. 
Proof. From Theorem 2.1, there exists a coupled coincidence point $(x, y) \in$ $X \times X$ of $T$ and $g$.

Now let $\left(x^{\prime}, y^{\prime}\right)$ be another coupled coincidence point of $T$ and $g$.

That is

$$
T\left(x^{\prime}, y^{\prime}\right)=g x^{\prime} \text { and } T\left(y^{\prime}, x^{\prime}\right)=g y^{\prime} .
$$

By additional assumption, there is $(u, v) \in X \times X$ such that $(T(u, v), T(v, u))$ is comparable to $(T(x, y), T(y, x))$ and $\left(T\left(x^{\prime}, y^{\prime}\right), T\left(y^{\prime}, x^{\prime}\right)\right)$.

Let $u_{0}=u, v_{0}=v, x_{0}=x, y_{0}=y, x_{0}^{\prime}=x^{\prime}$ and $y_{0}^{\prime}=y^{\prime}$.

Since $T(X \times X) \subseteq g(X)$, we can construct the sequences $\left\{g u_{n}\right\},\left\{g v_{n}\right\}$, $\left\{g x_{n}\right\},\left\{g y_{n}\right\},\left\{g x_{n}^{\prime}\right\}$ and $\left\{g y_{n}^{\prime}\right\}$ such that

$$
\begin{aligned}
& g u_{n+1}=T\left(u_{n}, v_{n}\right), g v_{n+1}=T\left(v_{n}, u_{n}\right) \\
& g x_{n+1}=T\left(x_{n}, y_{n}\right), g y_{n+1}=T\left(y_{n}, x_{n}\right) \\
& g x_{n+1}^{\prime}=T\left(x_{n}^{\prime}, y_{n}^{\prime}\right) \text { and } g y_{n+1}^{\prime}=T\left(y_{n}^{\prime}, x_{n}^{\prime}\right), n=0,1,2, \cdots
\end{aligned}
$$

Since $(g x, g y)=(T(x, y), T(y, x))=\left(g x_{1}, g y_{1}\right)$ and $(T(u, v), T(v, u))=\left(g u_{1}, g v_{1}\right)$ are comparable, then $g x \preceq g u_{1}$, and $g y \succeq g v_{1}$.

One can show that $g x \preceq g u_{n}$, and $g y \succeq g v_{n}$ for all $n$.

As in the Theorem 2.1, we conclude that $\left\{g u_{n+1}\right\} \rightarrow g x\left\{g v_{n+1}\right\} \rightarrow g y$.

Analogously, we can show that $\left\{g u_{n+1}\right\} \rightarrow g x^{\prime}\left\{g v_{n+1}\right\} \rightarrow g y^{\prime}$ in $(g(X), d)$. Since $g(X)$ is complete and $\left\{g u_{n+1}\right\}$ converges to $g x$ and $g x^{\prime}$, we get $g x=g x^{\prime}$.

Similarly $g y=g y^{\prime}$.

Thus if $(x, y)$ and $\left(x^{\prime}, y^{\prime}\right)$ are coupled coincidence points of $T$ and $g$, then

$$
T(x, y)=g x=g x^{\prime}=T\left(x^{\prime}, y^{\prime}\right)=\alpha, \text { say }
$$

and

$$
T(y, x)=g y=g y^{\prime}=T\left(y^{\prime}, x^{\prime}\right)=\beta, \text { say } .
$$

Since $(T, g)$ is $w$ - compatible, then

$$
g \alpha=g(g x)=g(T(x, y))=T(g x, g y)=T(\alpha, \beta)
$$

and

$$
g \beta=g(g y)=g(T(y, x))=T(g y, g x)=T(\beta, \alpha) .
$$

Hence the pair $(\alpha, \beta)$ is also coupled coincidence point of $T$ and $g$.

Thus we have

$$
g \alpha=g x \text { and } g \beta=g y .
$$

Therefore

$$
\alpha=g x=g \alpha=T(\alpha, \beta) \text { and } \beta=g y=g \beta=T(\beta, \alpha) .
$$


Thus $(\alpha, \beta)$ is a common coupled fixed point of $T$ and $g$.

Suppose $\left(\alpha^{1}, \beta^{1}\right)$ is another common coupled fixed point of $T$ and $g$.

Then $\alpha^{1}=T\left(\alpha^{1}, \beta^{1}\right)=g \alpha^{1}$ and $\beta^{1}=T\left(\beta^{1}, \alpha^{1}\right)=g \beta^{1}$.

Since $(\alpha, \beta)$ and $\left(\alpha^{1}, \beta^{1}\right)$ are coupled coincidence points of $T$ and $g$, it follows from (15) and (16) that $g \alpha=g \alpha^{1}$ and $g \beta=g \beta^{1}$ which implies that $\alpha=\alpha^{1}$ and $\beta=\beta^{1}$. Thus $(\alpha, \beta)$ is the unique common coupled fixed point of $T$ and $g$.

Example 2.3. Let $X=[0,1]$ and $d(x, y)=e^{|x-y|}$ for all $x, y \in X$ then $(X, d, \preceq)$ is a partially ordered complete multiplicative metric space. The ordering $\preceq$ is defined by $x \preceq y \Longleftrightarrow x \leq y$. Let $T: X \times X \rightarrow X$ and $g: X \rightarrow X$ be defined by $T(x, y)=\frac{x+y}{2}$ and $g(x)=x$. Then $T$ and $g$ satisfies all the conditions of Theorem 2.1 and Theorem 2.2. Clearly $(0,0)$ is the unique common coupled fixed point of $T$ and $g$.

Corollary 2.4. Let $(X, d, \preceq)$ be partially ordered complete multiplicative metric space and let $T: X \times X \rightarrow X$ be mapping satisfying

$$
\frac{1}{2} \min \{d(x, T(x, y)), d(u, T(u, v))\} \leq \max \{d(x, u), d(y, v)\}
$$

implies that

$$
d(T(x, y), T(u, v)) \leq \max \left\{\begin{array}{c}
d(x, u), d(y, v), d(x, T(x, y)), \\
d(y, T(y, x)), d(u, T(u, v)), d(v, T(v, u)), \\
\sqrt{d(x, T(u, v)) \cdot d(u, T(x, y))}, \\
\sqrt{d(y, T(v, u)) \cdot d(v, T(y, x))}
\end{array}\right\}
$$

for all $x, y, u, v \in X$, with $x \preceq u, y \succeq u$ and $\lambda \in\left(0, \frac{1}{2}\right)$

(2.4.2) (a) If a non- decreasing sequence $\left\{x_{n}\right\} \rightarrow x$ then $x_{n} \preceq x$ for all $n$,

(b) If a non- increasing sequence $\left\{y_{n}\right\} \rightarrow y$ then $y \preceq y_{n}$ for all $n$

If there exist $x_{0}, y_{0} \in X$ such that $x_{0} \preceq T\left(x_{0}, y_{0}\right)$ and $y_{0} \succeq T\left(y_{0}, x_{0}\right)$, then $T$ have a unique coupled fixed point in $X \times X$.

Proof. The proof of this corollary follows from Theorem 2.1 by taking $\psi(t)=t, \phi(t)=t^{1-\lambda}$ and $g(x)=x$.

Example 2.5. Let $X=[0,1]$ and $d(x, y)=2^{|x-y|}$ for all $x, y \in X$ then $(X, d, \preceq)$ is a partially ordered complete multiplicative metric space. The 
ordering $\preceq$ is defined by $x \preceq y \Longleftrightarrow x \leq y$. Let $T: X \times X \rightarrow X$ be defined by $T(x, y)=\frac{x+y}{4}$ and $\lambda=\frac{1}{4} \in\left(0, \frac{1}{2}\right)$. Consider

$$
\begin{aligned}
d(T(x, y), T(u, v)) & =2^{\left|\frac{x+y}{4}-\frac{u+v}{4}\right|} \\
& =2^{\left|\frac{x-u}{4}-\frac{v-y}{4}\right|} \\
& \leq \max \left\{2^{\left|\frac{x-u}{4}\right|,}, 2^{\left|\frac{y-v}{4}\right|,}\right\} \\
& =\max \left\{2^{\frac{1}{4}|x-u|,}, 2^{\frac{1}{4}|y-v|,}\right\} \\
& =\max \left\{2^{|x-u|}, 2^{|y-v|,}\right\}^{\frac{1}{4}}=\max \{d(x, u), d(y, v)\}^{\lambda} \\
& \leq \max \left\{\begin{array}{c}
d(x, u), d(y, v), d(x, T(x, y)), \\
d(y, x)), d(u, T(u, v)), d(v, T(v, u)), \\
\sqrt{d(x, T(u, v)) \cdot d(u, T(x, y))}, \\
\sqrt{d(y, T(v, u)) \cdot d(v, T(y, x))}
\end{array}\right\}
\end{aligned}
$$

Clearly $(0,0)$ is the unique coupled fixed point of $T$.

\section{References}

[1] M. Abbas, M. Alikhan and S. Radenović, Common coupled fixed point theorems in cone metric spaces for w-compatible mappings, Appl. Math. Comput., 217, No. 1 (2010), 195-202.

[2] M. Abbas, M. La Sen, and T. Nazir, Common fixed points of generalized rational type cocyclic mappings in multiplicative metric spaces, Descrete Dynamics in Nature and Society, 2015, Article ID 532725, 10 pages, doi: 10.1155/2015/532725.

[3] A.E. Bashirov, E.M. Kurpinar, A. Ozyapici, Multiplicative calculus and its applicatiopns, J. Math. Anal. Appl., 337 (2008), 36-48, doi: 10.1016/j.jmaa.2007.03.081.

[4] T.G. Bhaskar and V. Lakshmikantham, Fixed point theorems in partially ordered metric spaces and applications, Nonlinear Anal., 65 (2006), 1379-1393.

[5] X. He, M. Song, and D. Chen, Common fixed points for weak commutative mappings on a multiplicative metric space, Fixed Point Theory Appl., 48 (2014), 9 pages, doi: : 10.1186/1687-1812-2014-48.

[6] S.M. Kang, P. Nagpal, G. Sudhir Kumar, and Sanjay Kumar, Fixed Points for multiplicative expansive mappings in multiplicative metric spaces, Int. J. Math. Anal., 9, No. 39 (2015), 1939-1946.

[7] V. Lakshmikantham and Lj. Ćirić, Coupled fixed point theorems for nonlinear contractions in partially ordered metric spaces, Nonlinear Anal., 70 (2009), 4341-4349.

[8] M. Ozavsar and A. C. Cevikel, Fixed point of multiplicative contraction mappings on multiplicative metric spaces, ArXiv: 1205.5131v1 [math.GM], 2012.

[9] M. Sarwar and Badshah-e-Rome, Some unique fixed point theorems in multiplicative metric space, ArXiv: 1410.3384v2 [math.GM], 2014. 
[10] K.P.R. Rao, G.N.V. Kishore, and N. Van Luong, A unique common coupled fixed point theorem for four maps under $\psi-\phi$ contractive condition in partial metric spaces, $C U B O$, 14, No. 3 (2012), 115-127.

[11] K.P.R. Rao, G.N.V. Kishore, and V.C.C. Raju, A coupled fixed point theorem for two pairs of $w$-compatible maps using altering distance function in partial metric space, $J$. Adv. Res. Pure Math., 4, No. 4 (2012), 96-114.

[12] W. Shatanawi, B. Samet and M. Abbas, Coupled fixed point theorems for mixed monotone mappings in ordered partial metric spaces, Mathematical and Computer Modeling, 55 (2012), 680-687.

[13] T. Suzuki, A generalized Banach contraction principle which characterizes metric completeness, Proc. Amer. Math. Soc., 136 (2008), 1861-1869. 